\title{
Sarcopenic Obesity Exacerbates Health Risk in Older Adults More Than Sarcopenia or Obesity Alone
}

\author{
Brian Helsel ${ }^{1 *}$ and Joel E Williams ${ }^{2}$ \\ ${ }^{1} \mathrm{PhD}$ student in the Department of Public Health Sciences at Clemson University, USA \\ ${ }^{2}$ Associate Professor in the Department of Public Health Sciences at Clemson University, USA
}

Submission: March 06, 2018; Published: March 26, 2018

*Corresponding author: Brian Helsel, Department of Public Health Sciences, Clemson University, P.0. Box 340745, Clemson, SC 29634-0745, USA, Tel: (864) 656-1017; Fax: (864) 656-6227; Email: bhelsel@clemson.edu

\begin{abstract}
Sarcopenia, the age-related loss of muscle mass, can be diagnosed by having an appendicular skeletal muscle mass divided by height squared that is greater than two standard deviations (SD) or more lower than a mean derived from a healthy young reference sample. Obesity can be defined as having a body mass index greater than $30 \mathrm{~kg} / \mathrm{m}^{2}$. Sarcopenic obesity, if not treated, can lead to a greater risk of cardiovascular disease, metabolic disease, muscular deficiencies, and biomechanical problems.It is quickly becoming a serious issue in the United States and other developed countries. Sarcopenic obesity is more prevalent in older adults but is also observed among younger individuals who have low physical activity levels and a poor diet. This review examines cardiovascular disease risk, mobility issues, physical functioning, and quality of life in older adults with sarcopenic obesity and reviews some common intervention techniques that could lead to a healthier quality of life in individuals with sarcopenic obesity.
\end{abstract}

Keywords: Sarcopenic obesity; Sarcopenia; Obesity; Older adults; Elderly; Review

\section{Introduction}

Improved quality of medical services and the discovery of new methods to treat illnesses are allowing people to live longer than ever before. These advances in medical services can be largely attributed to the new developments made in technology, but the increasing focus on improving overall health and wellness also plays a large influential role in lifespan and quality of life. With the baby boom generation reaching older adulthood in the next few years, the number of expected individuals over the age of 65 years is expected to grow significantly. By the year 2020, an expected 55 million Americans will be over the age of 65 years and 6 million over the age of 85 years [1]. By the year 2030 , older adults in the United States over the age of 65 years could make up $20 \%$ of the population. It is important to educate older adults, their caretakers, and other professionals on the importance of using exercise and nutrition to increase the focus on health and wellness, decrease the risk of cardiovascular and metabolic disease, and improve overall quality of life.

One common disease that is increasingly prevalent in older adults is sarcopenia. Sarcopenia can be defined as the age related loss of muscle mass and is a clinical disorder that causes weakness, increased fatigability, and decreased physical performance [2]. It is more common in older adults $\geq 65$ years of age but can also be prevalent in younger adults who lead a sedentary lifestyle. Sedentary individuals at risk for sarcopenia should be evaluated and assigned to a strict exercise routine and encouraged to increase their activity level. Often times, a disorder like sarcopenia is accompanied by an increased amount of body fat mass due to low activity levels. The combination of sarcopenia with an increased body fat percentage or body mass index is called sarcopenic obesity. This mini review focuses on how to diagnose sarcopenic obesity, the health-related problems with the disease, and common intervention techniques that could lead to a higher quality of life in patients with sarcopenic obesity.

\section{Diagnosis of sarcopenic obesity}

Clinicians may diagnose sarcopenic obesity using criteria that are commonly used to measure sarcopenia and obesity separately [3]. Sarcopenia is frequently measured by appendicular skeletal muscle mass via dual energy x-ray absorptiometry (DXA) and visceral adiposity can be measured through computed tomography (CT), but definitions for sarcopenic obesity vary slightly. One research study defined sarcopenic obesity as having an appendicular skeletal mass divided by height squared (ASM/ Height $^{2}$ ) that is two standard deviations lower than a young reference population and a percentage of fat mass that is greater than the $60^{\text {th }}$ percentile for an age matched population [4]. 
Another study suggests appendicular skeletal mass adjusted for body mass is a better predictor for mobility and physical function [5]. Batsis et al. [6] found different prevalence rates based on using ASM when compared to ASM divided by body mass index (BMI) with data from the National Health and Nutrition Examination Survey (NHANES). The prevalence for sarcopenic obesity in a sample of 4,984 adults $\geq 60$ years in the NHANES 1999-2004 data was $24.4 \%$ and $22.7 \%$ using the ASM and ASM/BMI definitions, respectively. Further, the prevalence for sarcopenia was $29.9 \%$ and $23.0 \%$ using the ASM and ASM/BMI definitions, respectively. Kim et al. [7] used ASM/height ${ }^{2}$, ASM/ BMI, and thigh muscle cross-sectional area (tmCSA) adjusted by weight (tmCSA/weight) with sarcopenic obesity being defined as two standard deviations from a young reference group. Regardless of the definition used, the ratio of skeletal mass and adipose tissue may differ among body regions, so diagnosing sarcopenic obesity may need to utilize a variety of measures along with gender or age referents. Additionally, muscular strength may be an appropriate method for assessing sarcopenic obesity risk, but more research needs to be conducted on the relationship between muscle mass and strength in older adults and its association with various comorbidities.

\section{Sarcopenic obesity health outcomes}

Increased cardiovascular disease risk factors: Sarcopenic obesity may lead to higher cardiometabolic risk and has previously been associated with insulin resistance, prevalence of visceral obesity, and metabolic syndrome [7]. Stephen \& Janssen [8] investigated whether sarcopenia, obesity, or sarcopenic obesity served as a stronger predictor for cardiovascular disease risk in older adults $\geq 65$ years of age. Additionally, they examined the relationship between muscle mass and strength on cardiovascular risk. Researchers measured waist circumference, muscle mass, and hand grip strength in 3,366 patients and found that sarcopenic obese individuals had greater risk for cardiovascular disease when compared to patients with sarcopenia or obesity. Further, they found that muscle strength was a better predictor for cardiovascular disease risk than muscle mass with a $23 \%$ increased cardiovascular risk if a patient had a high waist circumference and low hand grip strength. Stephen \& Janssen [8] recommend that interventions should focus on decreasing abdominal fat and improving muscular strength to reduce the risk of cardiovascular disease and improve quality of life in the older adult population. Research has demonstrated that quadriceps muscle strength may be inversely related to several diseases, including diabetes, chronic heart disease, and chronic heart failure and that this negative relationship could be exacerbated with obesity, but whether muscle mass or strength is more associated with these conditions is less clear [1]. Elderly individuals who suffer from metabolic or cardiovascular diseases may have lower muscle mass, causing sarcopenia or sarcopenic obesity which can lead to disability, frailty, and activity of daily living impairments $[9,10]$.
Physical function, musculoskeletal deficiencies, and activities of daily living: Muscle dysfunction characterizes frailty syndrome, but the exact definition is unclear. In addition to low muscle mass and strength, poor endurance, balance, walking performance, or low activity may represent frailty syndrome. Frailty may be defined by disability, comorbidity, advanced old age, or cumulative decline across multiple systems. It may be triggered by lack of physical activity, inadequate nutrition, unhealthy environment, injuries, disease, age, or obesity. In addition to these factors, hormonal changes, proinflammatory responses, and skeletal, joint or neural deterioration may cause a decrease in strength and greater risk for sarcopenia [11]. One issue that arises with sarcopenic obesity and reduced muscular strength may be certain musculoskeletal and biomechanical deficiencies, including decreased bone mineral density, low muscular protein, and poor gait patterns. The lack of weight bearing activities in sarcopenic obese patients due to inactivity may increase the risk of a fracture or the development of osteoporosis because the bones are not being mechanically loaded and strengthened [12]. To increase bone strength, a patient must perform weight-bearing activities or resistance train. Sarcopenic obese patients may have a difficult time being physically active due to mobility issues. Mobility is a problem in older adults even without sarcopenia or sarcopenic obesity and higher adiposity or lower muscular strength only attenuates mobility. In a 6-year longitudinal study, Stenholm et al. [13] found that patients between the ages of 65 and 80 years who are obese and have lower muscular strength are at risk for a greater decline in walking speed and developing a mobility disability. Further, individuals with lower muscle mass may have a greater risk of functional impairment [14]. With decreased mobility and increased functional impairment, activities of daily living become more difficult in patients with sarcopenic obesity. Rolland et al. [15] found that when compared to the normal population, sarcopenic obese women had 2.60, 2.35, and 1.54 higher odds for difficulty climbing stairs, descending stairs, and other movements, respectively, concluding that the combination of sarcopenia and obesity significantly limited physical tasks. The loss of muscle mass due to aging is particularly detrimental to the lower extremity because it could lead to falls and fractures in the elderly, causing a decrease in daily living and independence.

\section{Exercise intervention}

Exercise interventions for sarcopenic obesity should aim to increase muscular mass and strength while decreasing adiposity. Previous research has used different intervention strategies including diet, exercise, and the combination of diet and exercise in order to assess which techniques would best improve physical function and decrease frailty in older adults. Villareal et al. [16] found that the combination of diet and exercise showed greater improvements in the physical performance test, $\mathrm{VO}_{2}$ peak, and functional status compared to the diet, exercise, and control groups. Exercise recommendations for sarcopenic obese 


\section{Current Research in Diabetes \& Obesity Journal}

individuals should include both aerobic and resistance training. The focus for aerobic exercise should be to reduce adiposity and achieve $\geq 5 \%$ weight loss in order to lower the risk for cardiovascular disease. Aerobic exercise should aim for a 500 to 750 kilocalorie per day energy deficit and 200 to 300 minutes of exercise per week [17]. Additionally, resistance training may be an effective weight management method in overweight or obese older adults because it can help prevent loss of lean muscle mass during diet intervention [18]. Fatouros et al. [19] suggests older adults who are overweight or obese could benefit greatly from two to three resistance training sessions per week to increase basal metabolic rate and total energy expenditure during activity that may lead to better weight management. Improvements in experiencing physical difficulties, physical capacity, muscle quality, fat-free mass, and gait speed may occur for sarcopenic patients in as few as 12 weeks of resistance training [20]. Other modalities, such as circuit training and aquatic exercise, may be effective in weight management, but more research needs to be conducted to see if either of these modalities can improve muscle mass and strength in older adults with sarcopenic obesity. Circuit and aquatic exercise training could be helpful for older adults who have problems with exercise adherence because these exercise modalities are often completed in group training sessions to increase the opportunity for developing relationships and utilize a wide variety of exercises to minimize boredom [21,22]. Additionally, aquatic exercise helps maximize safety and comfort, especially in patients with bone and joint disorders, allowing them to exercise for longer durations and more frequent [22].

\section{Conclusion}

Sarcopenic obesity raises serious concerns for health issues in older adults and sedentary individuals. The combined loss of muscle mass and obesity causes increased risk of cardiovascular and metabolic disease, decreases an individual's ability to carry out activities of daily living, and could impact overall health and wellness. Exercise and diet intervention has served as a beneficial method for preventing and treating sarcopenic obesity. Approaches to exercise should include a combination of cardiovascular and muscular training to increase lean muscle mass and decrease adiposity. Recommendations for appropriate frequency, intensity, duration, and mode of exercise in patients with sarcopenic obesity still need to be established in future research studies.

\section{Conflicts of Interest}

The author has no conflicts of interest to report. The author has not received payment or services from a third party for any aspect of the submitted work at any time. The authors' employer has not received payment or services from a third party for any aspect of the submitted work at any time. There are no other relationships or activities that readers could perceive to have influenced, or that give the appearance of potentially influencing, what is written in the submitted work.

\section{References}

1. Chen L, Nelson DR, Zhao Y, Cui Z, Johnston JA (2013) Relationship between muscle mass and muscle strength, and the impact of comorbidities: A population-based, cross-sectional study of older adults in the United States. BMC Geriatr 13: 74.

2. Batsis JA, Barre LK, Mackenzie TA, Pratt SI, Lopez Jimenez F, et al. (2013) Variation in the prevalence of sarcopenia and sarcopenic obesity in older adults associated with different research definitions: Dual-energy x-ray absorptiometry data from the national health and nutrition examination survey 1999-2004. J Am Geriatr Soc 61(6): 974980.

3. Kim TN, Park MS, Lim KI, Yang SJ, Yoo HJ, et al. (2011) Skeletal muscle mass to visceral fat area ratio is associated with metabolic syndrome and arterial stiffness: The Korean sarcopenic obesity study (KSOS). Diabetes Res Clin Pract 93(2): 285-291.

4. Schautz B, Later W, Heller M, Müller MJ, Bosy Westphal A (2012) Total and regional relationship between lean and fat mass with increasing adiposity -impact for the diagnosis of sarcopenic obesity. European Journal of Clinical Nutrition 66(12): 1356-1361.

5. Estrada M, Kleppinger A, Judge J0, Walsh SJ, Kuchel GA (2007) Functional impact of relative versus absolute sarcopenia in health older women. J Am Geriatr Soc 55(11): 1712-1719.

6. Batsis JA, Mackenzie TA, Jones JD, Lopez Jimenez F, Bartels SJ (2016) Sarcopenia, sarcopenic obesity, and inflammation: Results from the 1999-2004 National Health and Nutrition Examination Survey. Clinical Nutrition 35(6): 1472-1483.

7. Kim TN, Park MS, Lee EJ, Chung HS, Yoo HJ, et al. (2017) Comparisons of three different methods for defining sarcopenia: An aspect of cardiometabolic risk. Sci Rep 7: 1-9.

8. Stephen WC, Janssen I (2009) Sarcopenic-obesity and cardiovascular disease risk in the elderly. J Nutr Health Aging 13(5): 460-466.

9. Baumgartner RN, Wayne SJ, Waters DL, Janssen I, Gallagher D, et al. (2004) Sarcopenic obesity predicts instrumental activities of daily living disability in the elderly. Obes Res 12(12): 1995-2004.

10. Lee JS, Auyeung TW, Leung J, Kwok T, Leung PC, et al. (2011) Physical frailty in older adults is associated with metabolic and atherosclerotic risk factors and cognitive impairment independent of muscle mass. J Nutr Health Aging 15(10): 857-862.

11. Buch A, Carmeli E, Boker LC, Marcus Y, Shefer G, et al. (2016) Muscle function and fat content in relation to sarcopenia, obesity and frailty of old age -an overview. Experimental Gerontology 76: 25-32.

12. Verschueren S, Gielen E, O’Neill TW, Pye SR, Adams JE, et al. (2013) Sarcopenia and its relationship with bone mineral density in middleaged and elderly European men. Osteoporos Int 24(1): 87-98.

13. Stenholm S, Alley D, Bandinelli S, Griswold ME, Koskinen S, et al. (2009) The effect of obesity combined with low muscle strength on decline in mobility in older persons: results from the InCHIANTI study. International Journal of Obesity 33(6): 635-644.

14. Janssen I, Heymsfield SB, Ross R (2002) Low relative skeletal muscle mass (sarcopenia) in older persons is associated with functional impairment and physical disability. J Am Geriatr Soc 50(5): 889-896.

15. Rolland Y, Lauwers Cances V, Cristini C, Abellan van Kan G, Janssen I, et al. (2009) Difficulties with physical function associated with obesity, sarcopenia, and sarcopenic-obesity in community-dwelling elderly women: The EPIDOS (EPIDemiologie de l'OSteoporose) study. Am J Clin Nutr 89(6): 1895-1900.

16. Villareal DT, Chode S, Parimi N, Sinacore DR, Hilton T, et al. (2011) Weight loss, exercise, or both and physical function in obese older adults. N Engl J Med 364(13): 1218-1229. 


\section{Current Research in Diabetes \& Obesity Journal}

17. American Diabetes Association (2017) Obesity management for the treatment of type 2 diabetes. Diabetes Care 40(Suppl 1): S57-S63.

18. Avila JJ, Gutierres JA, Sheehy ME, Lofgren IE, Delmonico MJ (2010) Effect of moderate intensity resistance training during weight loss on body composition and physical performance in overweight older adults. Eur J Appl Physiol 109(3): 517-525.

19. Fatouros IG, Chatzinikolaou A, Tournis S, Nikolaidis MG, Jamurtas AZ, et al. (2009) Intensity of resistance exercise determines adipokine and resting energy expenditure responses in overweight elderly individuals. Diabetes Care 32(12): 2161-2167.
20. Liao CD, Tsauo JY, Lin LF, Huang SW, Ku JW, et al. (2017) Effects of elastic resistance exercise on body composition and physical capacity in older women with sarcopenic obesity: A CONSORT-compliant prospective randomized controlled trial. Medicine 96(23): e7115.

21. Bocalini DS, Lima LS, de Andrade S, Madureira A, Rica RL, et al. (2012) Effects of circuit-based exercise programs on the body composition of elderly obese women. Clin Interv Aging 7: 551-556.

22. Vasconcelos KSS, Dias JMD, Araújo MC, Pinheiro AC, Maia MM, et al. (2013) Land-based versus aquatic resistance therapeutic exercises for older women with sarcopenic obesity: Study protocol for a randomized controlled trial. Trials Journal 14: 296.

Your next submission with Juniper Publishers will reach you the below assets

- Quality Editorial service

- Swift Peer Review

- Reprints availability

- E-prints Service

- Manuscript Podcast for convenient understanding

- Global attainment for your research

- Manuscript accessibility in different formats

( Pdf, E-pub, Full Text, Audio)

- Unceasing customer service

Track the below URL for one-step submission https://juniperpublishers.com/online-submission.php 